\title{
The growth of the equidistance tendency over time
}

HOWARD LODGE 2 AND EUGENE R. WIST

FRANKLIN AND MARSHALL COLLEGE

To determine the manner in which the equidistance tendency (ET) varies as a function of time, $10 \mathrm{Ss}$ tracked the perceived position of a monocular test object viewed in a binocular field consisting of two rectangles which differed in perceived size and distance. Variables investigated were the temporal order of rectangle appearance and disappearance which determined whether or not opposing ETs existed, luminance and repetition interval of the $20 \mathrm{msec}$ test object. It was found that ET effects occur gradually over time, that opposing ETs retard the rate at which the test object changes its perceived locus, and that elapsed time rather than total observation time was critical in determining how great a change in perceived locus of the test object occurred. Implications of the se findings were discussed.

Gogel (1965) has provided a comprehensive review of the literature on the equidistance tendency (ET) and has demonstrated the usefulness of this tendency in accounting for perceptual phenomena such as Emmert's Law, the distorted room illusion and the moon illusion. Gogel defined ET as the tendency to perceive objects as equidistant in depth when effective cues to depth are minimal or absent.

This phenomena has been shown to occur when all the objects in the visual field are monocular or binocular (Gogel, 1956) or when some objects in the field are monocular and some binocular (Gogel, 1954; Wist, 1966). It has also been determined that the strength of ET varies inversely with the lateral separation of objects in the visual field (Gogel, 1956).

ET has also been implicated in perceived slants experiments. Bergman and Gibson (1959) and Gibson (1950) reported that without altering the physical angle of tilt, the perceived slant of a surface gradually decreased over time. Gogel (1954) demonstrated that the perceived depth interval between two objects differing in relative size decreases over time. Assuming that a texture gradient such as existed in the Bergman and Gibson (1959) and Gibson (1950) experiments constituted a relative size gradient, Gogel (1965) proposed that ET was the factor altering perceived slant in the texture gradient experiments. In an earlier paper, Harker (1962) proposed that ET was involved in producing changes in perceived slant in an experiment involving induced cyclorotation of the eyes.

The question of what the form of the relationship between changes in perceived relative depth and observation duration remains unanswered. The data of Clark (1953) support the possibility of a curvilinear relationship. His data suggest, if one assumes the equivalence of a texture and relative size gradient, that a critical duration exists beyond which further increases in duration would result in an increase rather than a decrease in the perceived relative depth of objects. The studies of Harker (1962), Gogel (1954), Bergman and Gibson (1959), and Gibson (1950) are compatible with the hypothesis that the relationship is monotonic.

It was the purpose of the present study to determine more directly the manner in which ET grows over time by employing an experimental technique which made possible continuous monitoring of the changes in depth position of a test stimulus under the influence of ET. One situation was designed to produce opposing ETs operating with respect to the test object, so that little or no change in the perceived distance of this object would occur over time. The other was designed to produce unopposed ETs so that relatively rapid and large changes in the perceived position of the test object would occur.

In addition, observation time was varied by presenting for a $4 \mathrm{~min}$ test period a test object $20 \mathrm{msec}$ in duration at repetition intervals of 50, 100, and $500 \mathrm{msec}$. Thus, after $1 \mathrm{~min}$ of elapsed time, cumulative observation time was either $17 \mathrm{sec}, 10 \mathrm{sec}$, or $2.3 \mathrm{sec}$, respectively, for the three repetition intervals. It was hypothesized that if ET grows as a function of observation time, repetition interval should affect the rate at which changes in the depth location of the test object occurred. If, on the other hand, elapsed time was the crucial variable, repetition interval should have no effect since elapsed time was equivalent $(4 \mathrm{~min}$ ) for the three repetition intervals.

\section{Subjects}

\section{METHOD}

The Ss were eight male college students and two male senior high school students between the ages of 17 and 21 years. All Ss possessed visual acuities of at least 20/20, stereoscopic acuities of at least $55 \mathrm{sec}$ of arc, and showed no evidence of abnormal phoria as measured with a Bausch and Lomb Orthorater. All Ss possessed normal color vision as measured by the Dvorine Pseuo Isochromatic color vision test.

\section{Apparatus}

The experiment was conducted in a light-tight visual alley $11 \mathrm{~m}$ long and $2.5 \mathrm{~m}$ wide. The $\mathrm{S}$ sat at one end of the alley in front of a panel contain- 


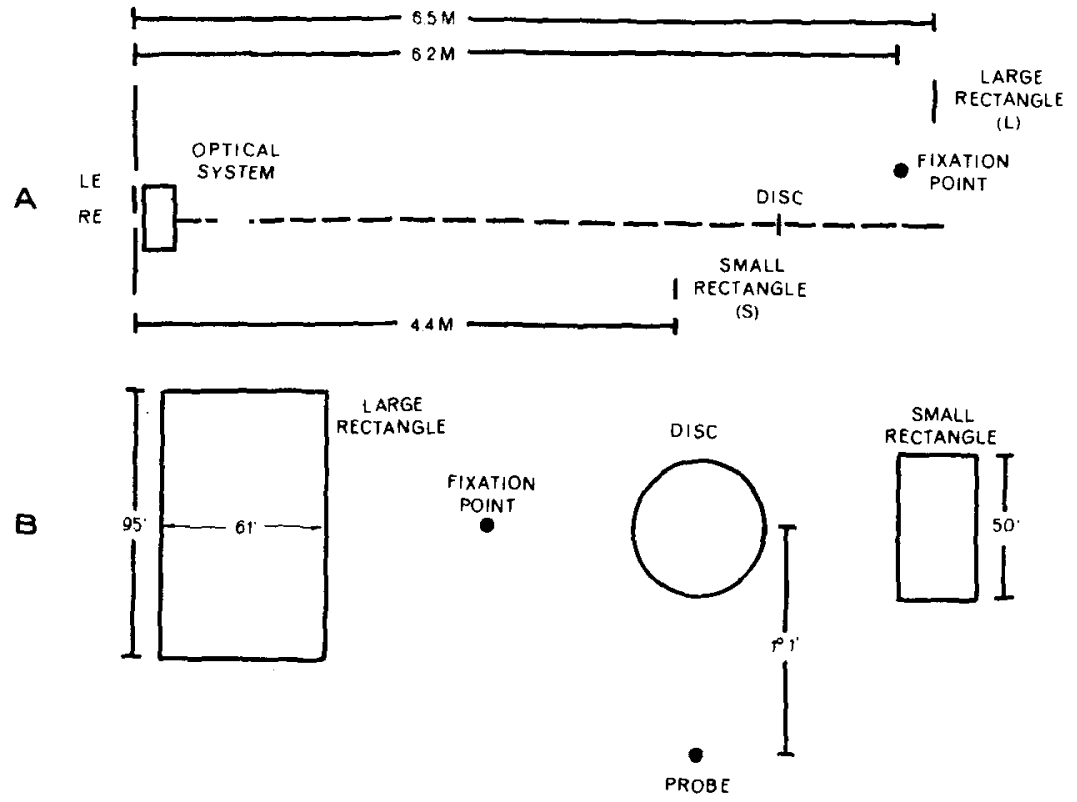

Fig. 1A. Overhead view of the visual alley showing the position of stimuli in relation to $S$. B. Frontal view of stimulus display with angular sizes and separations in min of visual arc.

ing two eye holes. Looking through these holes enabled $S$ to see five luminous stimuli at different distances (see Fig. 1a). The nearest stimulus was a small binocular rectangle at $4.4 \mathrm{~m}$. A binocular fixation point was located at $6.2 \mathrm{~m}$ and a large binocular rectangle was located at $6.56 \mathrm{~m}$ from $\mathrm{S}$. The lateral separations of these stimuli as well as their angular sizes are given in Fig. 1b. The disc was seen monocularly by the right eye and was generated by an optical system, in which light from a Sylvania R1131C glow modulator tube was passed through Kodak No. 96 neutral density filters of variable density, transilluminated a circular reticle which consisted of a small aperture in an otherwise opaque photographic negative, was collimated and then deflected into the eye by a beam splitter. This system allowed $S$ to view the image of the reticle down his median plane while viewing the binocular stimuli described above.

Duration of the disc and repetition interval were controlled by coupling a Kepco Model 400B regulated dc power supply with a flash generator (Roush \& Hamburger, 1948) which produced a train of voltage pulses which could be varied in duration and temporal spacing and which activated the glow modulator tube. This circuit and a similar optical system is described in more detail elsewhere (Wist \& Gogel, 1966).

The sources of illumination of the binocular rectangles were cool white, 12 in., $60 \mathrm{~W}$ fluorescent lamps, two per rectangle, enclosed in light-tight boxes. Variation in luminance was achieved by a system of ground glass and milk Lucite filters. Calibration was performed with a Spectra brightness spot meter, Model 1815-SB. The appropriate dimensions for these stimuli were achieved by masking the milk Lucite faces of the light boxes with construction board.

A $28 \mathrm{~V}$ incandescent lamp (GE $166 \mathrm{IF}$ ) was used as the luminous source for the fixation point. The intensity of this lamp was adjusted by means of a variable transformer. A small cubic construction board box enclosed the lamp. A $1 / 16$ in. hole in the frontal side of this box covered by a ground glass filter restricted the light output of the lamp to a small point in S's visual field.

The "probe"' (see Fig. 1b) located below the fixation point consisted of a substage microscope illuminator (American Science Center, Inc., Stock No. 15004) mounted on a small aluminum cart which could be moved on a two rail angle aluminum track along S's median plane. The light output of this source was reduced to a luminous point by a procedure identical to that used for the fixation point. The intensity of this source was also adjusted by means of a variable transformer. The intensities of both the fixation point and the probe were adjusted so that no surrounding "halo" was visible to S. Their luminance was approximately that of the rectangles.

During the experiment, S's task was to adjust the depth position of the continuously moving probe so that it appeared alternately just in front of or just behind the apparent position of the monocular disc in a manner analogous to that of the Békésy auditory threshold tracking task (1947). Movement of the probe along the track was accomplished by the use of an instantly reversible electric motor (Bodine Type KC1-23RM) connected by a system of pulleys, gears, and belts to the cart. The motor was activated by two switches, one which started and stopped the probe and another which controlled its direction of 
movement. Continuous monitoring of the position of the probe was achieved by feeding the resistance changes of a 10 turn potentiometer which was mechanically linked to the pulley and gear system into a resistance-programmed power supply (Harrison Labs Model 6206A). The power supply produced a voltage which was linearly related to the physical position of the probe. This voltage was fed into an Esterline Angus recording voltmeter which provided a permanent record of S's tracking performance.

\section{Procedure}

Tasks. Subjects performed two tasks, a brightness matching task and the tracking task mentioned earlier. The first task involved matching the brightness of the disc and the brightness of the small rectangle to that of the large rectangle by the method of adjustment so that in spite of the area and shape differences between these three stimuli, each appeared to be equal in brightness. (Two exceptions to this procedure are described later.) Subjects performed this task at the beginning of each experimental and control session. The second task involved activating the motor by means of a switch and thus causing the probe to move continuously in depth along the median plane. The $S$ was instructed to reverse the direction of movement of the probe by means of a second switch when the probe appeared just in front of or just behind the perceived position of the flashing disc. Thus, S's task was a dynamic one, in which he tracked changes in the perceived depth position of the disc by means of the probe. This procedure will subsequently be referred to as "tracking" the disc.

Experimental Design. Three independent variables were investigated in this study. The $20 \mathrm{msec}$ disc was presented at repetition intervals of 50 , 100, and $500 \mathrm{msec}$ as measured from disc onset to onset. Four temporal rectangle sequences were presented: Large rectangle alone, Both rectangles, Small rectangle alone (LBS); Large rectangle alone, Small rectangle alone (LS); Small rectangle alone, Both rectangles, Large rectangle alone (SBL); and Small rectangle alone, Large rectangle alone (SL).

For sequences LBS and SBL two ETs operated during the period in which both rectangles were simultaneously present, one between the small rectangle and the disc, and one between the large rectangle and the disc.4 These two tendencies were predicted to have opposing effects on the perceived depth location of the disc, with the former making the disc appear closer and the latter making the disc appear farther from S. Since no opposition of ETs existed for the LS and SL sequences, relatively rapid shifts in the depth position of the disc were predicted when one and then the other member of the pair were presented in succession. Furthermore, these latter two conditions provided a check on the adequacy of the tracking task as a measure of changes in perceived distance. If only the first two sequences had been administered and the tracking data indicated no shifts in perceived depth of the disc, we could not have been sure that this was not due to the insensitivity of the tracking task to changes in the perceived depth location of the disc.

The third independent variable was the luminance of the disc. All 10 ss were run under one condition in which the brightness of the disc and the small rectangle were matched to the intensity of a $2 \mathrm{ft}-\mathrm{L}$ large rectangle. In addition, six of the Ss ran under another condition in which matching was performed to a $1 \mathrm{ft}-\mathrm{L}$ large rectangle. For the remaining four Ss, the additional condition involved matching the disc and small rectangle to a $.5 \mathrm{ft}-\mathrm{L}$ large rectangle. For all three luminances, however, the tracking task was performed with a luminance of $2 \mathrm{ft}-\mathrm{L}$ for both rectangles. Thus, each $S$ was run under three repetition intervals, four temporal sequences of rectangle presentation, and two conditions of disc brightness for a total of 24 conditions. These conditions were presented in a counterbalanced order over $151-\mathrm{h}$ sessions. In addition, two practice sessions were given. During the first the brightness matching task was performed and, during the second, the tracking task was performed.

Light Adaptation. At the beginning of each session, Ss received $5 \mathrm{~min}$ of light adaptation facing a $2 \mathrm{ft}-\mathrm{L}$ homogeneous screen $60^{\circ}$ high and $80^{\circ}$ wide. During this period $S$ was given instructions for the performance of the tasks. At the end of this period, s performed the brightness matching task, after which he received an additional $3 \mathrm{~min}$ of light adaptation. At the end of this period, he began the tracking task. For experimental conditions LBS and SBL, $S$ tracked the perceived position of the disc for $1 \mathrm{~min}$ with the initial rectangle illuminated and then tracked the disc for an additional $4 \mathrm{~min}$ with the second rectangle also present, after which the initial rectangle was turned off and tracking continued for $1 \mathrm{~min}$ with the second rectangle only. An experimental session consisted of four such 6 min tracking sets with $3 \mathrm{~min}$ of light adaptation to screen between each set.

For the control sessions, LS and SL, S tracked the disc for $1 \mathrm{~min}$ with the initial rectangle illuminated. This rectangle was then turned off and tracking continued for an additional $5 \mathrm{~min}$ with only the second rectangle present. Control sessions were $1-1 / 2 \mathrm{~h}$ long and consisted of eight such 6 min tracking sets with $3 \mathrm{~min}$ of light adaptation between each set.

\section{RESULTS}

The raw data consisted of S's tracking performance recorded on the recording voltmeter for each of the $6 \mathrm{~min}$ tracking sets. Figure 2 is an example of a record for one of the Ss for condition LBS when 


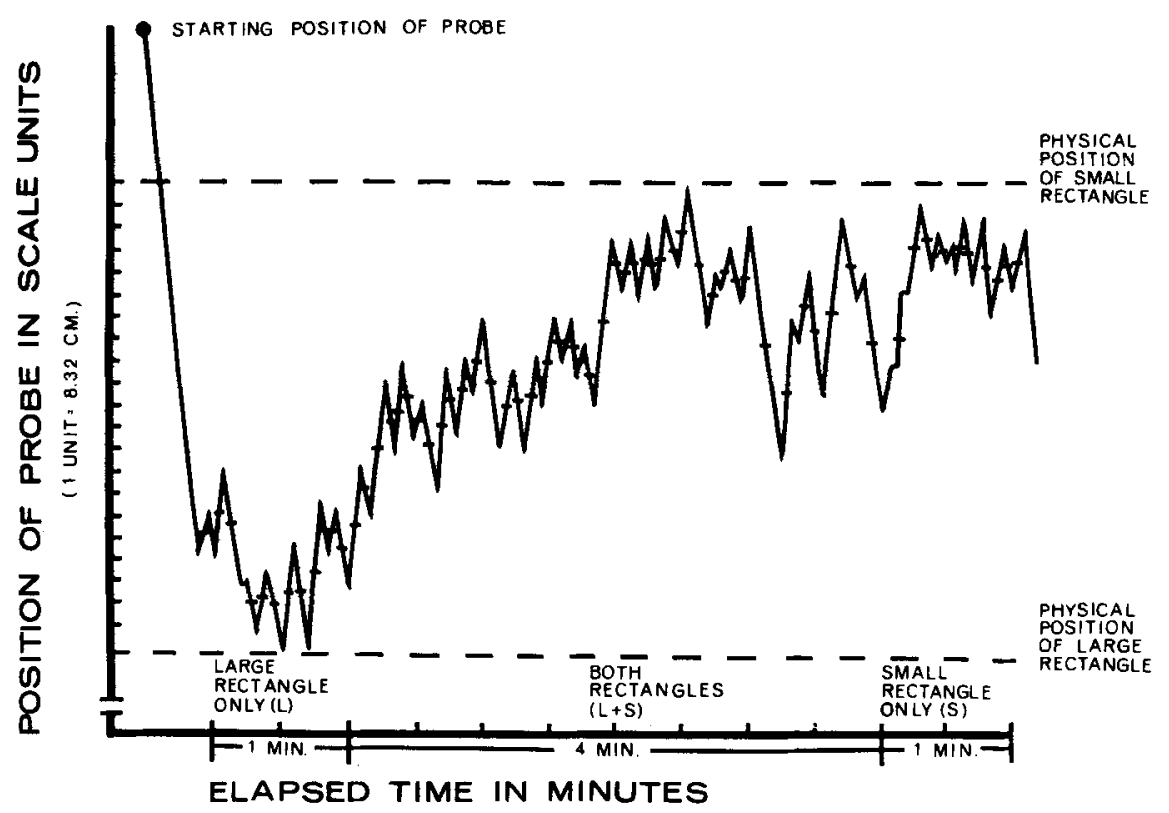

Fig. 2. An example of a record of the "tracking" of the perceived position of the disc of one $s$. For the 6 min period on the abscissa, the disc was tracked with a repetition interval of 500 msec. Its brightness was matched to equal that of the 2 it-L large rectangle. The sequence of rectangle presentation was LBS. the repetition interval of the disc was $500 \mathrm{msec}$ and its intensity was matched to the $2 \mathrm{ft}-\mathrm{L}$ large rectangle. In this figure, the physical positions of the small and large rectangles is indicated by the dashed lines at right angles to the ordinate. The starting position of the probe was in front of the small rectangle. When the large rectangle was turned on, the probe moved back toward this rectangle until $S$ operated the reversing switch. The successive reversals recorded in this initial $1 \mathrm{~min}$ segment of the record constitute a measure of the perceived position of the disc when only the large rectangle was present. The tracking for the next $4 \mathrm{~min}$ represents the perceived position of the disc after the small rectangle was turned on, and the final $1 \mathrm{~min}$ segment represents the perceived position of the disc when only the small rectangle was presented. The dashes locating the midpoints of each reversal of the probe were used in determining the perceived depth position of the disc for the $6 \mathrm{~min}$ period represented on the abscissa. A mean depth position for the initial $1 \mathrm{~min}$ period when only the large rectangle was present was calculated by summing the number of volts in scale units represented by the 11 midpoints in this period and dividing by 11 . Similarly, mean depth positions were calculated for each of the following eight $.5 \mathrm{~min}$ periods, and finally for the last $1 \mathrm{~min}$ period in which the small rectangle only was present. This procedure was used in calculating the perceived position of the disc for all experimental and control conditions. The resulting mean perceived depth positions in volts were then converted into centimeter equivalents. For this conversion, $0 \mathrm{~cm}$ represented the physical location of the closest (smaller) rectangle and $216 \mathrm{~cm}$ repre- sented the physical location of the farther (larger) rectangle.

Figure 3 presents the data for the $2 \mathrm{ft}-\mathrm{L}$ condition. The mean depth position of the disc in centimeters for all $10 \mathrm{Ss}$ is plotted as a function of elapsed time for the four temporal sequences of rectangle presentation. The point on the abscissa labeled $1.0 \mathrm{~min}$ represents the initial depth location of the disc, while the point labeled $6.0 \mathrm{~min}$ represents the terminal perceived position of the disc. The intervening $.5 \mathrm{~min}$ points represent the periods in which either both rectangles were present simultaneously (conditions SBL and LBS) or in which the second rectangle was presents only (rectangle $L$ for condition SL, and rectangle $\mathbf{S}$ for condition LS). Since repetition interval was not found to have a significant effect on the depth position of the disc for this condition, the data for repetition intervals were pooled.

Figure 3 shows that for condition LS that when the small rectangle followed the large rectangle, the disc shifted in depth position toward $S$ over time and, when the large rectangle followed the small rectangle (condition SL), the disc shifted away from $S$ over time. Furthermore, it can be seen that when opposing ETs existed (conditions LBS and SBL), smaller shifts in the depth position of the disc occurred than when no opposing ETs existed (conditions LS and SL). Analyses of variance of the data of this graph resulted in statistically significant $F$ ratios for temporal sequence and elapsed time. 5 In addition, Ss differed significantly and a significant subjects by temporal sequence interaction was obtained. All of these effects were significant at $p<$ .01. Although the appearance of the graph seems to suggest a temporal sequence by elapsed time 


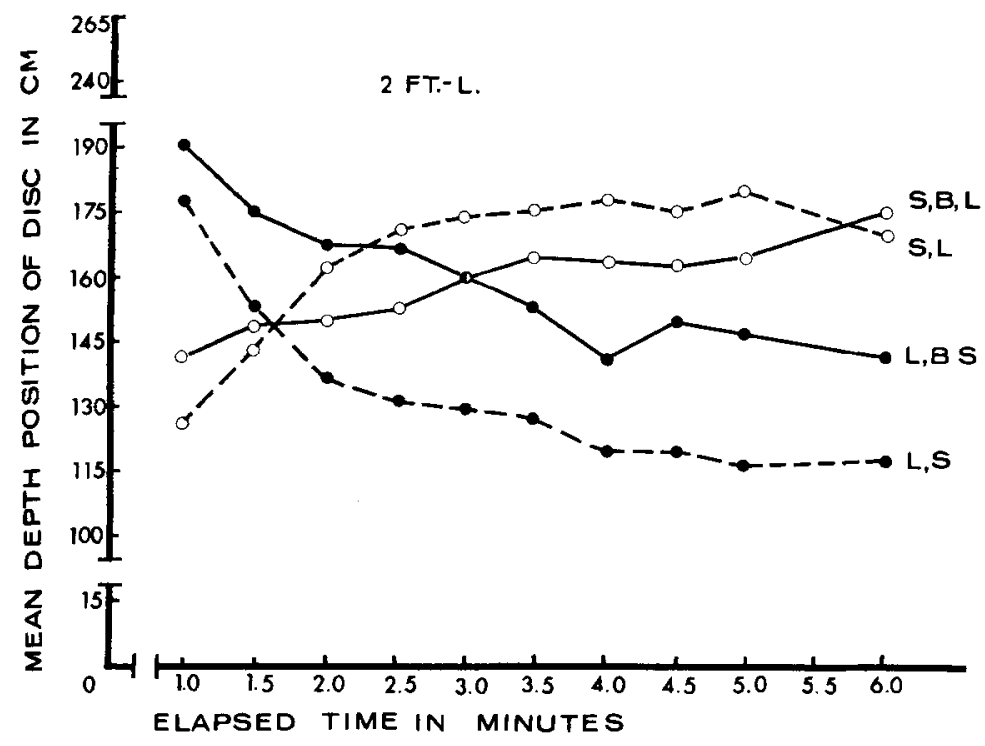

Fig. 3. Mean depth position of the disc for $10 \mathrm{Ss}$ for the four rectangle présentation sequences as a function of elapsed time. The brightness of the disc was matched to that of the 2 It-L large rectangle. Data for the three repetition intervals are pooled.

interaction, statistical significance was not achieved for this effect. However, when the SL, SBL, LS, and LBS conditions were analyzed separately, a significant temporal sequence by elapsed time interaction $(p<.01)$ was obtained for the first pair of conditions, but not for the last pair.

In general, the results obtained for the 1 and $.5 \mathrm{ft}-\mathrm{L}$ conditions were similar to those of the 2 $\mathrm{ft}-\mathrm{L}$ condition described above. The curves obtained for the SL and SBL conditions shown in Figs. 4 and 5 are quite like those in Fig. 2. Major differences were found with respect to the LS and LBS conditions. For the $1 \mathrm{ft}-\mathrm{L}$ condition (Fig. 4), the main effect of repetition interval was significant for the LS condition $(p<.01)$. The $50 \mathrm{msec}$ repetition interval showed a significantly greater change over the $5 \mathrm{~min}$ period than the 100 and $500 \mathrm{msec}$ curves.

For the $.5 \mathrm{ft}-\mathrm{L}$ condition (Fig. 5), no statistically significant difference was obtained between the LS and LBS conditions, indicating that at the lowest disc luminance, the amount of shift in the perceived position of the disc was about the same regardless of whether or not opposing ETs were present.

\section{DISCUSSION}

At least four important findings are evident in the results of this study: (1) The ET between two stimuli in the visual field does grow monotonically as a function of time in the absence of additional stimuli. This effect was shown by both the SL and LS conditions. This finding is consistent with those of Gibson (1950) and Bergman and Gibson (1959) in which the perceived slant of textured surfaces were investigated, and with Gogel's (1965) interpretation of the results of these studies. This finding (or any of the others) is not consistent with data supporting the
Fig. 4. Mean depth position of the disc for 6 Ss for the four rectangle presentation sequences as a function of elapsed time. The brightness of the disc was matched to that of a $1 \mathrm{ft}-\mathrm{L}$ large rectangle. Data for the three repetition intervals are pooled except for condition LS where a significant effect of repetition interval on depth position was obtained.

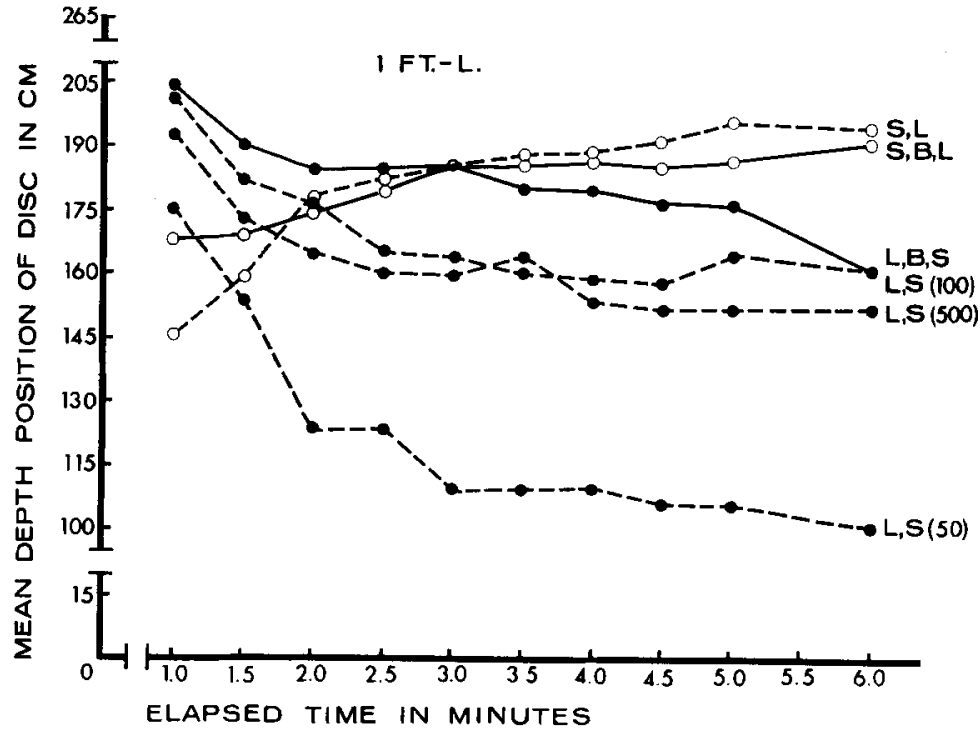




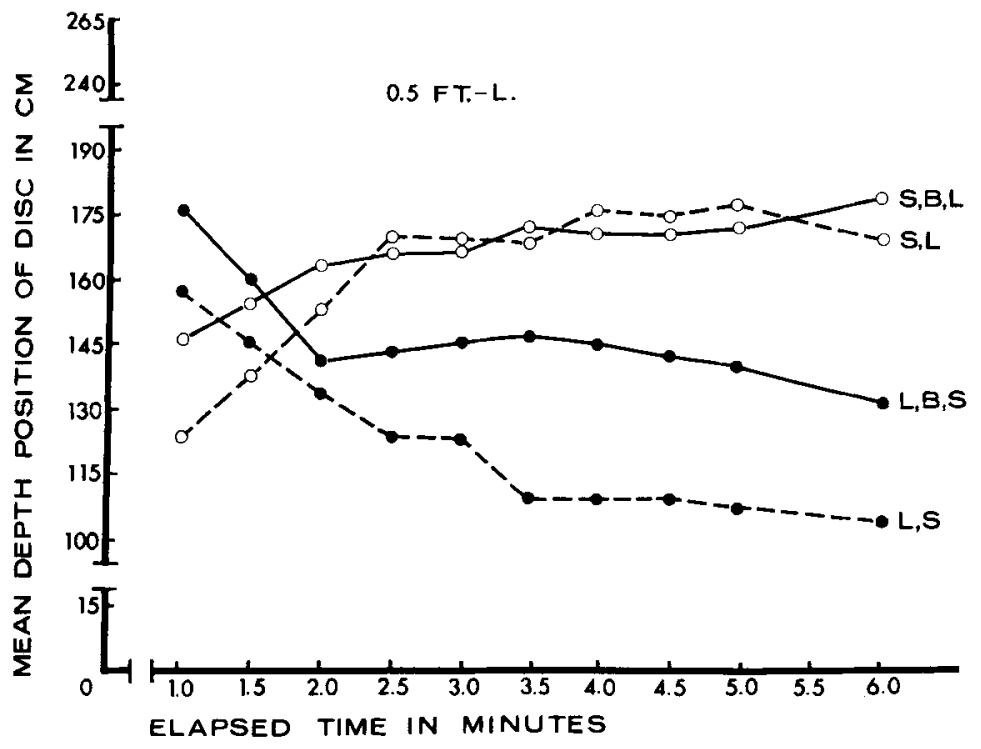

Fig. 5. Mean depth position of the disc for $4 \mathrm{Ss}$ for the four rectangle presentation sequences as a function of elapsed time. The brightness of the disc was matched to that of a $5 \mathrm{ft}-\mathrm{L}$ large rectangle. Data for the three repetition intervals are pooled.

possibility of a curvilinear relationship (Clark, 1953). (2) When opposing but unequal ETs exist in the visual field, their effect is to retard the rate at which a test object under their influence changes its perceived position. This was especially evident for the SBL condition when compared to the LS condition. (3) The luminance of the test stimuli can modify the above effects. It was found that when the test stimulus (the disc) had a luminance one-half that of the other stimuli ( $1 \mathrm{ft}-\mathrm{L}$ ), the shortest repetition interval resulted in a more rapid change in perceived position of the test object when no opposing ETs were present (Fig. 3). At the lowest luminance (.5 $\mathrm{ft}-\mathrm{L}$ ) where the test stimulus was one-fourth the luminance of the other stimuli, changes in the perceived position of this stimulus occurred less rapidly and were of a smaller extent. This result suggests that perceived brightness (or luminance) is an important cue determining the strength of the ET. (4) At least for the sort of stimulus situation investigated here, the rate at which even a test object not under the influence of opposing ETs changes in perceived position is slow, a matter of minutes rather than seconds. Furthermore, elapsed time rather than total observation time of the test object appeared to be the more important variable. Repetition interval failed to have an effect except for one of the 12 possible combinations of rectangle sequence and luminance. Yet over the $6 \mathrm{~min}$ test period, the $20 \mathrm{msec}$ disc was seen for widely varying total times: $102 \mathrm{sec}, 60 \mathrm{sec}$, and $14 \mathrm{sec}$, for the 50, 100 , and $500 \mathrm{msec}$ repetition intervals, respectively. From this one can conclude that the perceptual "adaptation" responsible for the shifts in depth position of the disc is not based on a mechanism which is very closely linked to stimulus variables such as rate of stimulation and total energy over time. The importance of elapsed time suggests a more central mechanism, one for which perceptual set or attentional variables are more relevant. The fact that pilot studies revealed a large effect of fixation on the rate at which the disc shifted in perceived position is consistent with such a mechanism. As fixation shifts across the visual display the relative contributions of the various stimuli to the resulting perceived position of the disc varied. The position of the fixation point chosen for the present study was such that $S$ could "attend to" both rectangles while fixating. Such effects are to be predicted on the basis of previous research on the effect of lateral separation of stimuli on the strength of ET (Gogel, 1956), for, as fixation shifts, the lateral separation of stimuli changes with respect to the direction of fixation. Subjects were instructed to maintain fixation at all times, but, there was no way of determining the extent to which fixation shifts occurred during the course of a session. Subjects did report when questioned that they tended to shift fixation momentarily when a new stimulus was added to the display. With somewhat larger lateral separations it would be possible to replicate this study while recording eye movements. In this way the effects of fixation shifts could be directly investigated.

An important methodological problem has implications for the generality of the findings in this study. It concerns the effect of the Békésy measurement technique itself. To what extent did an ET operate between the probe and the disc? If the probe appreciably "attracted" the disc, then the perceived depth location of the latter would vary as the perceived position of the former varied during the course of the measurement period. Two lines of evidence suggest that this was not the case. First of all, during the initial $1 \mathrm{~min}$ period of tracking before the introduction of the second rectangle, 
there was no evidence that the starting position of the probe had any effect on the perceived position of the disc as determined by taking the means of the midpoints of probe reversals. If the disc were being influenced by the probe, such an effect should have been found. Secondly, some data were obtained from some Ss at the end of the experiment in which the probe was absent. Subjects reported changes in the perceived position of the disc verbally by indicating such changes in terms of the number of multiples of rectangle height by which the disc appeared either in front of or behind the rectangle. This was done at intervals over $6 \mathrm{~min}$ periods. The results were consistent with those reported here. Thus, it would appear that the probe per se did not have an appreciable effect on the perceived position of the disc. Even if it did have some effect not detectable by the above procedures, this effect was not large enough to override the effects of temporal sequence and luminance.

In any case, the data of this study support the conclusion that the growth of the equidistance tendency over elapsed time is a monotonic one when measured during a period in which a new stimulus is added that disrupts the previous balance of ETs. This change is a relatively slow one, taking place over minutes rather than seconds. However, the rate of change is very probably a function of the degree to which the stimulus added to the visual field modifies the balance of ETs which existed initially. One methodological caution is apparent from the findings of this study. In studies on the ET, viewing time must be carefully controlled. Successive measures of depth location after the introduction of a new object in the visual field will vary systematically as a function of time.

\section{References}

Bekésy, G. A new audiometer. Acta Oto-laryngol., 1947, 35, 411422 .
Bergman, R., \& Gibson, J. J. The negative after effect of the perception of a surface slanted in the third dimension. Amer. J. Psychol., 1959, 72, 364-373.

Clark, C. W. Exposure time and the perception of slant. Paper read at Canad. Psychol. Assoc., June, 1953.

Gibson, J. J. The perception of visual surfaces. Amer. J. Psychol, $1950,63,367-384$.

Gogel, W. C. Perception of the relative distance position of objects as a function of other objects in the field. J. exp. Psychol., $1954,47,335-342$.

Gogel, W. C. The tendency to see objects as equidistant and its inverse relation to lateral separation. Psychol. Monogr.. 1956. 70, Whole No. 411 .

Gogel, W. C. Equidistance tendency and its consequences. Psychol. Bull. 1965, 64, 153-163.

Harker, G. S. Apparent fronts parallel plane, stereoscopic correspondence, and induced cyclotorsion of the eyes. Percept. mot. Skills, 1962, 14, 75-87.

Roush, R., \& Hamburger, F., Jr. Light-flash generator. Electronics, $1948,21,100-102$.

Wist, E. R., \& Gogel, W. C. The effect of interocular delay and repetition interval on depth perception. Vision Res., 1966, 6, 325-334.

Wist, E. R. The influence of the equidistance tendency on depth shifts resulting from an interocular delay in stimulation. Paper read at Eastern Psychol. Assoc. meetings, New York City, April, 1966.

\section{Notes}

1. This study is based on the honors thesis of the first author. The second author is primarily responsible for the present form of the manuscript. The research was supported in part by NSF URP Grant No. 10362-01 and NIH Grant No. 05528-02 to the second author.

2. Now at Psychology Department, Indiana University, Bloomington, Indiana.

3. The authors wish to thank Walter Martin, Director of the Franklin and Marshall Computer Center for writing the program for the statistical analyses and for carrying them out.

4. It is true, of course, that a third equidistance tendency operated between the two rectangles, but because retinal disparity existed for these stimuli, the strength of the equidistance tendency between them was small relative to the equidistance tendencies between each of the binocular stimuli and the monocular disc. (See Gogel, 1956)

5. Treatments by Treatments by Subjects analyses of variance as described by Lindquist, 1956, p. 237.

(Accepted for publication October 15, 1967.) 